\title{
Heavy flavour production in ATLAS
}

\author{
Brad Abbott ${ }^{a, *}$ \\ ${ }^{a}$ University of Oklahoma, \\ Norman, Oklahoma, USA \\ E-mail: abbott@ou.edu
}

The associated production of vector boson with quarkonia is a key observable for understanding the quarkonium production mechanisms, including the separation of single and double parton scattering components. Using data collected by the ATLAS experiment, located at the LHC, measurements of prompt and non-prompt $J / \psi$ and $\psi(2 S)$ mesons with $p_{\mathrm{T}}$ between $60-360 \mathrm{GeV}$ is reported at $\sqrt{s}=13 \mathrm{TeV}$ in $p p$ collisions. A measurement of the production of a prompt $J / \psi$ in association with a $W^{ \pm}$boson is presented at $\sqrt{s}=8 \mathrm{TeV}$ in $p p$ collisions.

40th International Conference on High Energy physics - ICHEP2020

July 28 - August 6, 2020

Prague, Czech Republic (virtual meeting)

\footnotetext{
${ }^{*}$ On behalf of the ATLAS Collaboration
} 


\section{Charmonium high $p_{\mathrm{T}}$ production cross sections at $\mathbf{1 3} \mathrm{TeV}$}

Charmonium production can provide important insight into QCD near the perturbative and non-perturbative boundary. Increasing the $p_{\mathrm{T}}{ }^{1}$ range for the production of quarkonium states is important since high $p_{\mathrm{T}}$ behavior may help discriminate between different theoretical models. Previous ATLAS measurements primarily required dimuon triggers which limited the $p_{\mathrm{T}}$ range to be less than $100 \mathrm{GeV}$. By using single muon triggers and the full Run 2 dataset, measurements at high $p_{\mathrm{T}}(60-360 \mathrm{GeV})$ are possible.

The dataset consists of $139 \mathrm{fb}^{-1}$ of data collected at ATLAS [1], located at the LHC, at $\sqrt{s}=13$ $\mathrm{TeV}$ [2]. All measurements are made in the dimuon decay channel and all events are weighted to take into account the acceptance, reconstruction efficiencies and trigger efficiencies. Using a two-dimensional unbinned maximum-likelihood fit to the dimuon invariant mass and pseudo-proper time, the yield of prompt and non-prompt charmonium components are extracted.
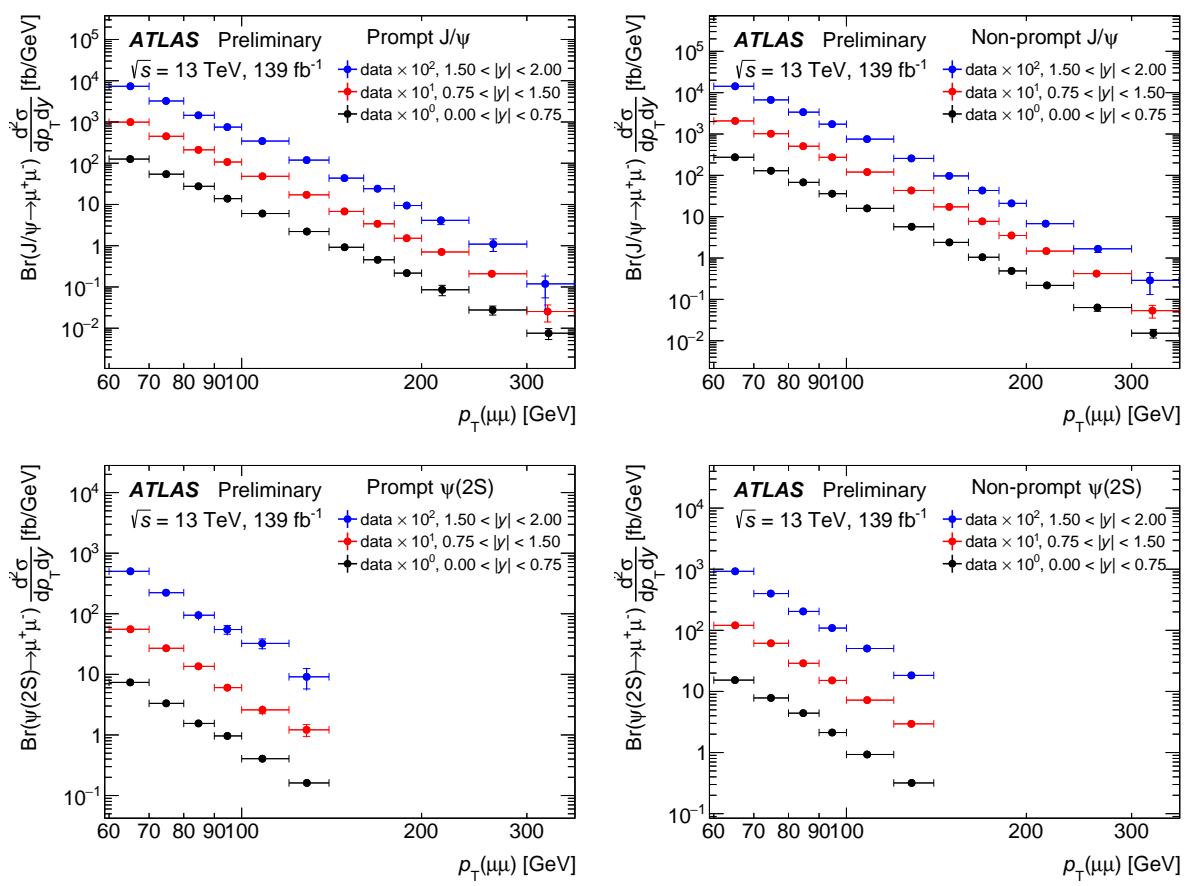

Figure 1: Differential cross sections of prompt and non-prompt production of $J / \psi$ mesons (top) and $\psi(2 S)$ mesons (bottom) [2].

The results shown in Figures 1-4 indicate that the $p_{\mathrm{T}}$ dependence for both prompt and nonprompt differential distributions are similar. The non-prompt fractions are approximately constant as a function of $p_{\mathrm{T}}$ for both $J / \psi$ and $\psi(2 S)$ mesons. The FONLL [3, 4] predictions are consistent with the data in the lower $p_{\mathrm{T}}$ range for non-prompt production but exceed the experimental results at larger $p_{\mathrm{T}}$. For further details about these results see Ref. [2].

\footnotetext{
${ }^{1}$ ATLAS uses a right-handed coordinate system with its origin at the nominal interaction point (IP) in the centre of the detector and the $z$-axis along the beam pipe. The $x$-axis points from the IP to the centre of the LHC ring, and the $y$-axis points upward. Cylindrical coordinates $(r, \phi)$ are used in the transverse plane, $\phi$ being the azimuthal angle around the $z$-axis. The pseudorapidity is defined in terms of the polar angle $\theta$ as $\eta=-\ln \tan (\theta / 2)$.
} 

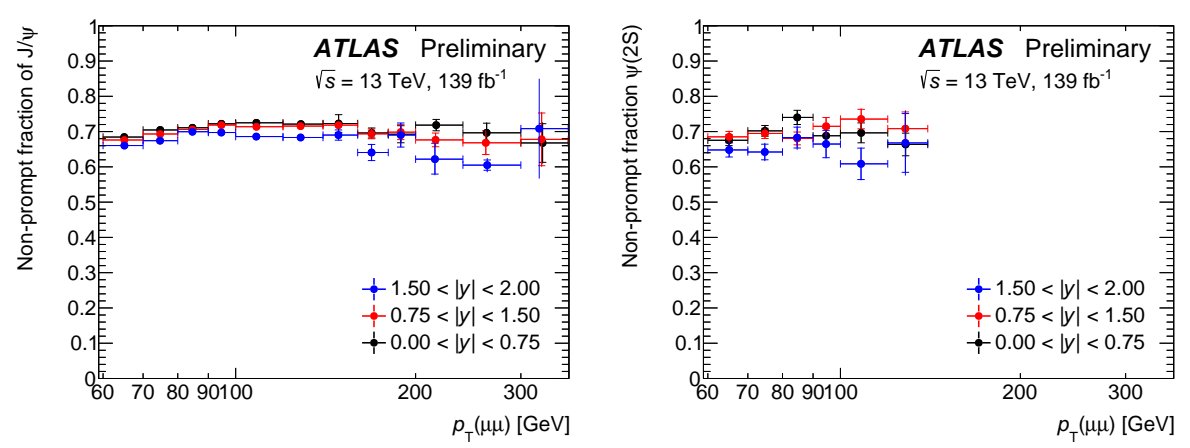

Figure 2: Non-prompt production fraction for $J / \psi$ (left) and $\psi(2 S)$ (right) [2].
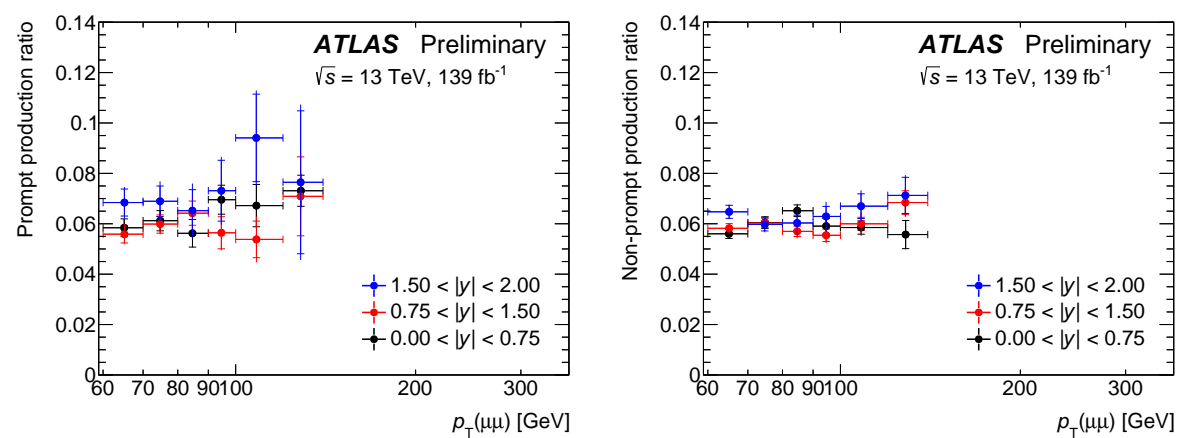

Figure 3: Ratio of $\psi(2 S)$ production with respect to $J / \psi$ for prompt (left) and non-prompt (right) production mechanisms [2].

\section{2. $J / \psi$ production in association with a $W^{ \pm}$boson at $8 \mathrm{TeV}$}

Charmonium production mechanisms in hadronic collisions are not completely understood since the relative contribution of color singlet to color octet production is unknown. By requiring an associated object, such as a $W^{ \pm}$boson, it is possible to filter the various color singlet/color octet diagrams. Additionally, the contribution of double parton scattering (DPS) versus single parton scattering (SPS) is unknown and measuring $\Delta \phi$ between the $J / \psi$ and $W^{ \pm}$can probe the relative contributions.

The dataset consists of $20.3 \mathrm{fb}^{-1}$ of data collected at $\sqrt{s}=8 \mathrm{TeV}$ [5]. All events were selected using a non-prescaled single muon trigger. An inclusive $W^{ \pm}$sample is collected by requiring a prompt, isolated muon with $p_{\mathrm{T}}>25 \mathrm{GeV}$, missing transverse momentum $>25 \mathrm{GeV}$ and the $W^{ \pm}$ boson transverse mass $>40 \mathrm{GeV}$.

In addition, if an event has two muons which satisfy a $J / \psi$ muon criterion, this event is defined as the associated $J / \psi+W^{ \pm}$sample. All events in the associated sample are weighted to take into account the acceptance and reconstruction efficiencies of the two muons from the $J / \psi$. A two-dimensional unbinned maximum-likelihood fit to the dimuon invariant mass and pseudo-proper time is used to extract the prompt charmonium components. After the fit is performed, the sPlot tool [6] is used to extract per-event weights which allow the azimuthal opening angle $\Delta \phi\left(J / \psi, W^{ \pm}\right)$to 

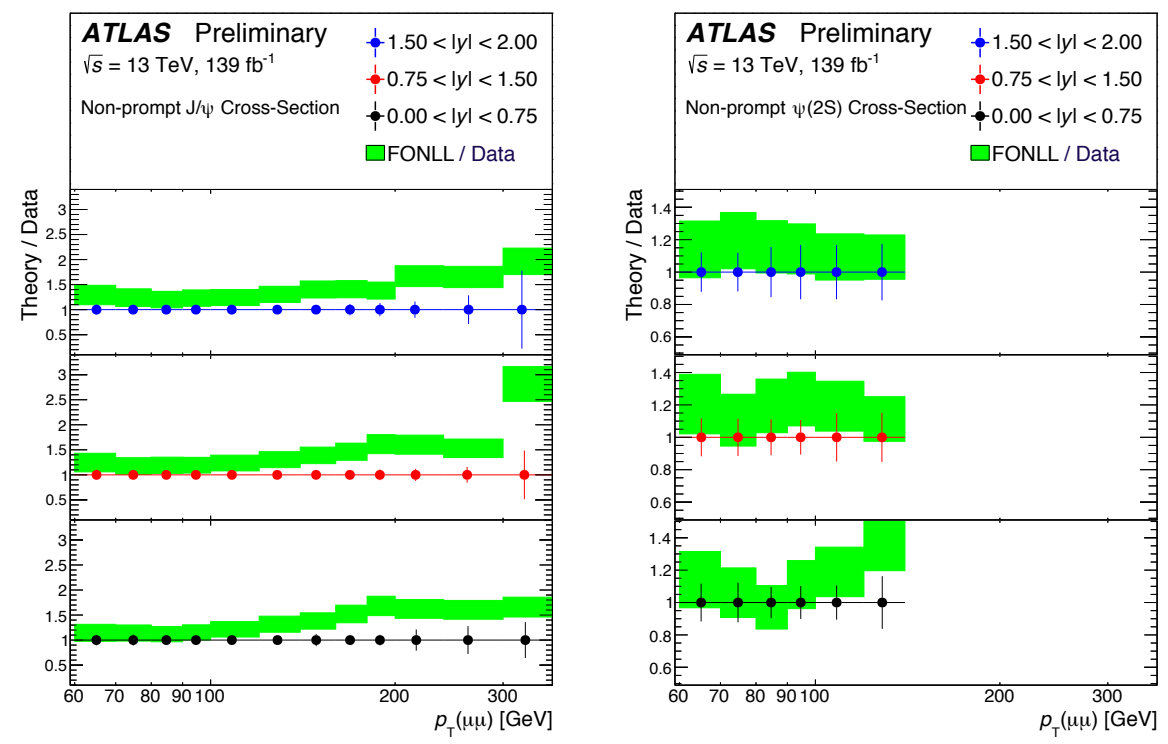

Figure 4: Ratio of the FONLL prediction to the measured differential cross sections for non-prompt $J / \psi$ (left) and $\psi(2 S)$ (right) mesons [2].

be measured. Figure 5 shows the sPlot-weighted opening angle $\Delta \phi\left(J / \psi, W^{ \pm}\right)$for prompt $J / \psi+W^{ \pm}$ candidates.

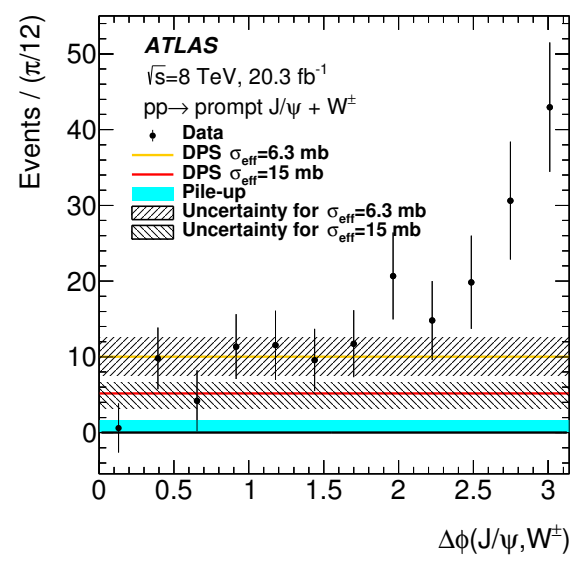

Figure 5: The sPlot-weighted opening angle $\Delta \phi\left(J / \psi, W^{ \pm}\right)$for prompt $J / \psi+W^{ \pm}$candidates [5].

Based on the assumption that the two hard scatters are uncorrelated, the probability that a $J / \psi$ is produced by a second hard process in an event containing a $W^{ \pm}$boson can be written as

$$
P_{J / \psi \mid W}^{i j}=\frac{\sigma_{J / \psi}^{i j}}{\sigma_{\mathrm{eff}}},
$$


where $\sigma_{J / \psi}^{i j}$ is the cross-section for $J / \psi$ production in the appropriate $p_{\mathrm{T}}(i)$ and rapidity $(j)$ interval and $\sigma_{\text {eff }}$ is the effective transverse overlap area of the interacting partons. Since $\sigma_{\text {eff }}$ may not be process-independent, two different choices of $\sigma_{\text {eff }}$ are considered. The data show that both choices of $\sigma_{\text {eff }}$ is consistent with the data at low $\Delta \phi$. The data is also consistent with both SPS and DPS contributions.

A fully corrected inclusive production cross-section ratio, in which the $J / \psi$ acceptance and the unknown $J / \psi$ spin-alignment are taken into account, is given by

$$
R_{J / \psi}^{\text {incl }}=\frac{\sigma_{\text {incl }}(p p \rightarrow J / \psi+W)}{\sigma(p p \rightarrow W)} \cdot \mathcal{B}(J / \psi \rightarrow \mu \mu)=\frac{1}{N(W)} \sum_{p_{\mathrm{T}} \text { bins }}\left[N^{\text {eff }+ \text { acc }}(J / \psi+W)-N_{\text {pile-up }}\right]
$$

where $N^{\text {eff }+ \text { acc }}(J / \psi+W)$ is the background subtracted yield of prompt $J / \psi+W$ events after $J / \psi$ acceptance corrections and efficiency corrections for the $J / \psi$ decay muons, and $N_{\text {pile-up is the }}$ expected number of pile-up events in the full range of $J / \psi$ decay phase space. The result is

$$
R_{J / \psi}^{\mathrm{incl}}=\left(5.3 \pm 0.7 \pm 0.8_{-0.7}^{+1.5}\right) \times 10^{-6},
$$

where the first uncertainty is statistical, the second systematic and the third uncertainty is due to the choice of spin-alignment scenario.

Additional measurements are found by subtracting the estimated DPS contribution in each $p_{\mathrm{T}}$ and rapidity interval from the inclusive cross-section ratio,

$$
R_{J / \psi}^{\mathrm{DPSsub}}=\left(3.6 \pm 0.7_{-1.0}^{+1.1+0.7}+1.5\right) \times 10^{-6}, \quad\left[\sigma_{\mathrm{eff}}=15_{-4.2}^{+5.8} \mathrm{mb}\right]
$$

and

$$
R_{J / \psi}^{\mathrm{DPSsub}}=\left(1.3 \pm 0.7 \pm 1.5_{-0.7}^{+1.5}\right) \times 10^{-6}, \quad\left[\sigma_{\mathrm{eff}}=6.3 \pm 1.9 \mathrm{mb}\right]
$$

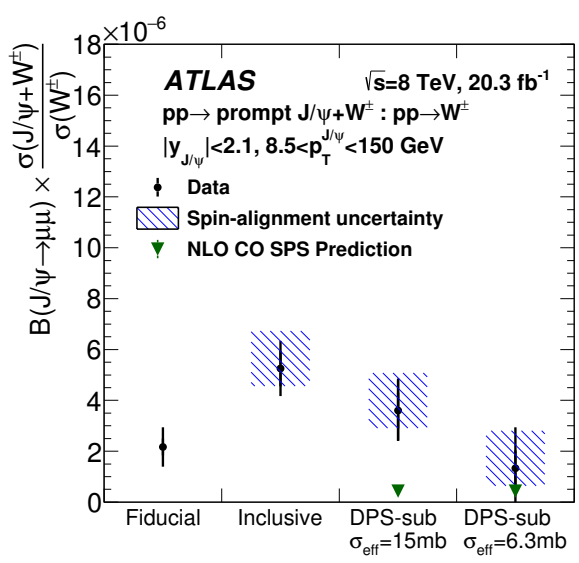

Figure 6: Production cross section ratios for the measurement in the fiducial volume (bin 1), the ratio corrected for acceptance effects (bin 2) and the ratio corrected for double parton scattering for two different

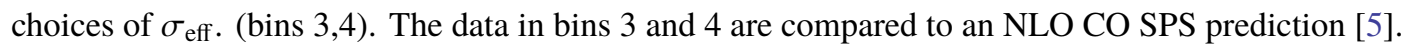



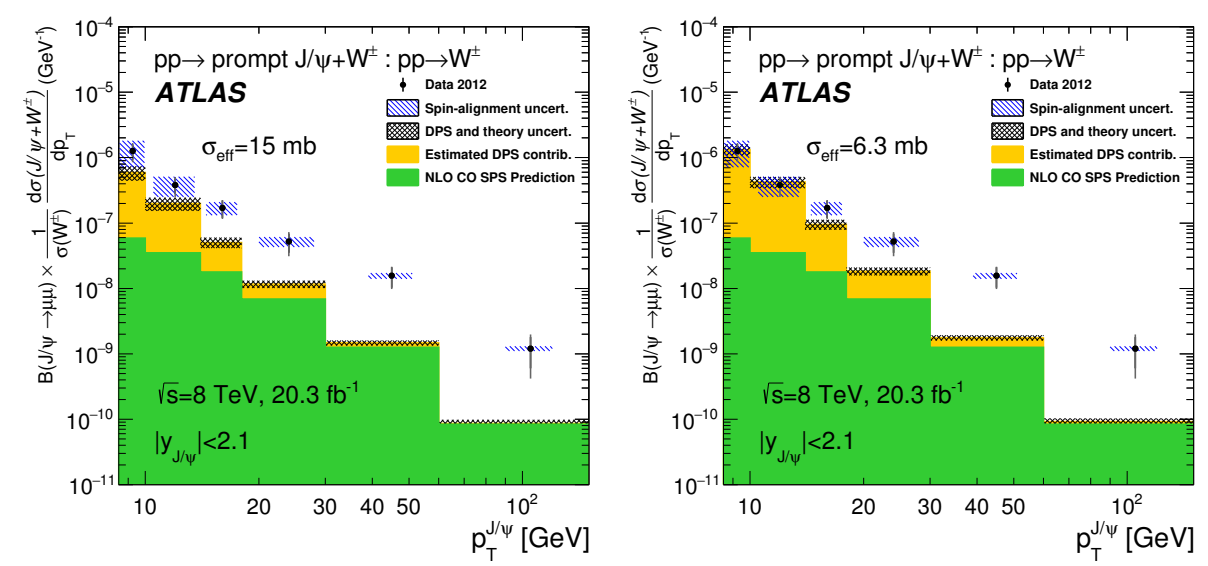

Figure 7: The inclusive differential cross-section ratio compared to theory in six different $p_{\mathrm{T}}^{J / \psi}$ regions for $\sigma_{\text {eff }}=15 \mathrm{mb}$ (left) and $\sigma_{\text {eff }}=6.3 \mathrm{mb}$ (right) [5].

Figures 6 and 7 show the production cross section ratio and differential cross section ratio for two different choices of $\sigma_{\text {eff }}$ compared to a NLO CO SPS prediction [7, 8]. A smaller value of $\sigma_{\text {eff }}$ is preferred however neither value of $\sigma_{\text {eff }}$ can describe the $p_{\mathrm{T}}^{J / \psi}$ dependence. For further details about these results see Ref. [5].

\section{References}

[1] ATLAS Collaboration, "The ATLAS Experiment at the CERN Large Hadron Collider", 2008 JINST 3 S08003

[2] ATLAS Collaboration, "Measurement of the production cross section of $J / \psi$ and $\psi(2 S)$ mesons at high transverse momentum in pp collisions at $\sqrt{s}=13 \mathrm{TeV}$ with the ATLAS detector", ATLAS-CONF-2019-047, http://cdsweb.cern.ch/record/2693955

[3] M. Cacciari et al. "Theoretical predictions for charm and bottom production at the LHC", JHEP 10 (2012) 137.

[4] M. Cacciari, S. Frixione and P. Nason, "The $p_{T}$ spectrum in heavy flavor photoproduction", JHEP 0103 (2001) 006

[5] ATLAS Collaboration, "Measurement of $J / \psi$ production in association with a $W^{ \pm}$boson in $p p$ data at $8 \mathrm{TeV}$ ", JHEP 01 (2020) 095

[6] M. Pivk and F. R. Le Diberder, "SPlot: A Statistical tool to unfold data distributions", Nucl. Instrum. Meth. A 555 (2005) 356

[7] P. Cho and A. K. Leibovich, “Color-octet quarkonia production”, Phys. Rev. D 53 (1996) 150

[8] P. Cho and A. K. Leibovich, "Color-octet quarkonia production II", Phys. Rev. D 53 (1996) 6203 